\title{
Cognitive zonal fusion biopsy of the prostate: Original technique between target and saturation
}

\author{
Andrea B. Galosi ${ }^{1}$, Guevar Maselli ${ }^{3}$, Giulia Sbrollini ${ }^{1}$, Gaetano Donatelli ${ }^{3}$, Lorenzo Montesi ${ }^{1}$, \\ Matteo Tallè ${ }^{1}$, Rodolfo Montironi ${ }^{2}$ \\ ${ }^{1}$ Department of Clinical Sciences and Dentistry, Urology Clinic, \\ 2 Pathology, Az. Ospedaliera Polytechnic University of Marche, Ancona, Italy; \\ ${ }^{3}$ Division of Urology, ASUR Marche, Fermo, Italy.
}

\begin{abstract}
Summary We describe our experience in prostate biopsy using a new standardized cognitive fusion techniques, that we call "cognitive zonal fusion biopsy". This new technique is based on two operative options: the first based on target biopsies, the Cognitive Target Biopsy (CTB) if the same target was detected with transrectal ultrasound (TRUS) and multiparametric magnetic resonance (mpMRI); the second based on saturation biopsies, the Zonal Saturation Biopsy (ZSB) on anatomical zone/s containing the region of interest if the same target was not evident with TRUS and MRI. We evaluated results of our technique compared to standard biopsy in order to identify clinically relevant prostate cancer.

Methods: This is a single-center prospective study conducted in 58 pts: 25 biopsy-naïve, 25 with previous negative biopsy and in 8 with cancer in active surveillance. Based on mpMRI and transrectal ultrasonography (TRUS), all patients were scheduled for standard 12-core TRUS-guided biopsy. If mpMRI was suggestive or positive (PI-RADS 3, 4 or 5): patients underwent additional targeted 2 to 6 cores using cognitive zonal fusion technique.

Results: $31 / 58$ (53.4\%) patients had a cancer. Our technique detected $80.6 \%$ (25 of 31) with clinically significant prostate cancer, leading to detection of insignificant cancer in $20 \%$. Using standard mapping in MR negative areas we found 5 clinically significant cancer and 4 not significant cancers. MRI cancer detection rate was 18/31 (58.1\%), and 9/18 (50\%) in high grade tumors. Therefore MRI missed $50 \%$ of high grade cancers. The mean number of cores taken with cognitive zonal fusion biopsy was 6.1 (2-17), in addition biopsy sampling was done outside the ROI areas. Overall 15.4 cores (12-22) were taken. Cancer amount in Zonal Biopsy was larger than 7.3 $\mathrm{mm}$ (1-54.5) in comparison with $5.2 \mathrm{~mm}(1-23.5)$ in standard mapping. Largest percentage of cancer involvement with cognitive zonal fusion technique was detected in 19.4\% vs $15.9 \%$. Conclusions: Cognitive Zonal Saturation Biopsies should be used to reduce operator variability of cognitive fusion biopsy in addition to standard biopsy. Cognitive zonal biopsy based on mpMRI findings identifies clinically relevant prostate in $80 \%$, has larger cancer extension in fusion biopsies than in random biopsies, and reduce the number of cores if compared to saturation biopsy.
\end{abstract}

KEY WORDS: Prosate neoplasms; Prostate biopsy; Fusion imaging; Ultrasound; Prostatectomy.

Submitted 15 November 2016; Accepted 15 December 2016

\section{INTRODUCTION}

Magnetic Resonance Imaging (MRI) is now becoming the imaging of choice for men with suspected prostate cancer, in particular index lesion detected by multiparametric MR (mpMR) may help us in risk-stratification. Several approaches have been explored to improve the accuracy of image-guided targeted prostate biopsy, including inbore MRI-guided, cognitive fusion, and MRI/transrectal ultrasound fusion-guided biopsy (1). Image-guided biopsy on MRI-target rather than ultrasound-target improves amount of tumor in biopsy cores and reduces the number of cores to harvest diagnosis (2). However, it has been recently observed that standard 12-cores biopsy still maintains the same cancer detection rate (53.1\%) if compared to targeted in bore MRI procedures, even in the rate of clinically significant cancer (3).

The debate is open on the question that we can't answer right now is whether we should omit the systematic conventional 12-core biopsies in patients with negative mpMR.

We attempt to advance our extensive experience in prostate biopsy using a new standardized cognitive fusion techniques, that we call "cognitive zonal fusion biopsy". In the standard cognitive fusion biopsy, the target is based on target biopsy of the the area supposed by operator after reading the MRI. This technique is extremely dependent on operator, his experience and imaging interpretation. Our technique, cognitive zonal fusion, is based on the following principles: 1) ultrasound target biopsy using endfire probe (4), that is performed if a target could be clearly showed comparing transrectal ultrasonography (TRUS) and MRI, 2) saturation biopsy principle, that is the standard method suggested by guideline to systematic detect or exclude prostate cancer (5). Therefore applying this principles to daily practice, if a target lesion is evident comparing TRUS and MRI, the saturation biopsy is limited to the target using 2-4 cores: this is called Cognitive Target Biopsy (CTB). Whereas, in prostate without target lesion detectable by TRUS if compared to MRI, the saturation biopsy is extended to anatomical zone or zones described by MR: this is called Zonal Saturation Biopsy (ZTB). Thus we supposed to reduce variability linked to cognitive fusion biopsy. Based on this hypothesis, aim of 
the study is to describe the technique and evaluate the benefits of the cognitive zonal fusion biopsy in addition to standard biopsy in order to identify clinically relevant prostate cancer. Furthermore, we evaluated cancer detection rate of Cognitive Target Biopsy which is used in case of a suspicious finding on ultrasound associated to mpMR suspicious finding, and Zonal Saturation Biopsy which is performed when there is no suspicious finding on ultrasound associated to mPMRI results.

\section{Materials AND Methods}

This is a single-center prospective study conducted in 58 pts (mean age of 64 years, 46-79): 25 biopsy-naïve men with elevated levels of PSA, 25 men with previous negative biopsy (including 8 with Atypical Small Acinar Proliferation) and 8 prostate cancer in active surveillance. Digital rectal examination was positive in 9 men (15\%), mean serum PSA was $6.8 \mathrm{ng} / \mathrm{ml}(0.7-22.6)$. Thirty-three patients (56.9\%) had already had 1 up to 6 previous prostate biopsy (mean 1.6). Patients features are listed in Table 1.

\section{Table 1.}

Patients characteristics at fusion biopsy.

\begin{tabular}{|lc|}
\hline & Tot \\
\hline Patients & 58 \\
\hline Median age (years) & $64(46-79)$ \\
\hline Naive biopsy for elevated PSA & $25(43 \%)$ \\
\hline N pts with previous biopsy & $25(43 \%)$ \\
\hline$\cdot$ Pts in active surveillance & $8(13.8 \%)$ \\
\hline Mean number of previuos biopsies & $1.6(1-6)$ \\
\hline DRE + & $9(15 \%)$ \\
\hline PSA (mean, ng/ml) & $6.8(0.7-22)$ \\
\hline Patients with positive mpMRI (PIRADS 3-5) & $53(91 \%)$ \\
\hline PI-RADS 3 & 36 \\
\hline PI-RADS 4-5 & 17 \\
\hline Patients with negative mpMRI (PIRADS 1-2) & $5(8 \%)$ \\
\hline
\end{tabular}

Table 2.

Biopsy features after Cognitive Zonal Fusion biopsy.

\begin{tabular}{|lc|}
\hline & Tot \\
\hline $\mathbf{N}^{\circ}$ of pts with cancer in biopsy & $31(53,4 \%)$ \\
\hline$\cdot$ High grade ca. & $18 / 31(58 \%)$ \\
\hline Clinically significant ca. & $25 / 31(80,6 \%)$ \\
\hline$\cdot$ Not significant ca. & $6 / 31(19,3 \%)$ \\
\hline $\mathrm{mpMRI} \mathrm{detection} \mathrm{rate} \mathrm{for} \mathrm{all} \mathrm{ca.}$ & $18 / 31(58,1 \%)$ \\
\hline $\mathrm{mpMRI}$ detection for High grade ca. & $9 / 18(50 \%)$ \\
\hline $\mathrm{mpMRI}$ sensitivity & $18 / 22$ \\
\hline Ca. detection in the CFB & $14 / 25(56 \%)$ \\
\hline Ca. detection outside CFB & $5 / 13(38.5 \%)$ \\
\hline Ca. detection in PI-RADS 4/5 & $8 / 17(47 \%)$ \\
\hline Ca. detection in PI-RADS 3 & $16 / 36(44 \%)$ \\
\hline $\mathrm{N}^{\circ}$ of cores in CTB (mean) & $6.1(2-17)$ \\
\hline Histology core length (mean, cm) & $1.5(0.8-1.9)$ \\
\hline ·large cancer inside CFB & $19.44 \%(0-67.53 \%)$ \\
\hline ·large cancer outside CFB & $15.9 \%(0-50.7 \%)$ \\
\hline
\end{tabular}

All the participants underwent contrast enhanced mpMRI in several centers with different degree of experience in public and private Italian hospitals, however all with adequate technical instrumentations most of them using 1.5 Tesla. Prostate Imaging Report and Data System (PIRADS) and zonal anatomical identification of the Region of Interest (ROI) were available in all reports. If mpMRI was negative (PI-RADS 1-2), subjects received at least a standard 12-core TRUS-guided biopsy plus additional cores according to prostate volume up to 20 cores. If mpMRI was suggestive (PI-RADS 3 ) or positive of cancer (PI-RADS 4 or 5) underwent 12-core biopsy plus targeted 2 to 6 cores using cognitive zonal fusion technique.

Cognitive Target Biopsy (CTB) on ROI (Region of interest) was done on a target if a correspondence was found between MRI and ultrasound (Figure 1). In addition the CTB was done if a anatomic ultrasound marker was close to the ROI identified by MR images (example: cyst or calcification, Figure 2).

If any ultrasound lesion or anatomic marker was detected comparing ultrasound with MR imaging, or a missing correspondence was found, the Zonal Saturation Biopsy (ZSB) was done. Zonal Saturation was performed using an uniform spatial distribution of random biopsies (2 up to 6) to cover the anatomic zone identified by MRI.

Based on MR images, prostate gland was divided in 14 areas according to standardized MRI prostate reporting scheme according to PI-RADS reporting (2), therefore a whole random sampling of the ROI was done including one or more anatomic zone which harbors the suspected MRI area. Also additional cores in adjacent areas can be taken in large gland (volume $\geq 60 \mathrm{cc}$ ). Trained operator in TRUS imaging and MR imaging performed all procedures. Definition of clinically significant tumor based on biopsy and radical prostatectomy specimen was defined by a dedicated uro-pathologist. Definition of clinically significant tumor based on biopsy was based on: tumor grade (Gleason Grading 3+4, or WHO Grade grouping $\geq 2$ ), tumor involvement $>50 \%$ of each biopsy core, cancer extension in more than 2 different anatomic zones, tumor marker above the upper limit (PSA > $10 \mathrm{ng} / \mathrm{ml}$ and/or PSA-Density > 0.15). Perineural and ductal invasion detected on biopsy specimens was recorded by a expert uro-pathologist. Definition of clinically significant tumor based on radical prostatectomy specimen: for grading (any Gleason Grade pattern 4, or Grade grouping 2), tumor volume $>0.50 \mathrm{cc}$ based on stereological (i.e. grid) method on histologic slides (6), local invasion of seminal vesicle (T3b) or extracapsular disease (T3a).

\section{Results}

In our study, 31/58 (53.4\%) patients had a cancer, 18/31 had high grade tumors (Gleason pattern 4 or 5 ). Twentyfive of 31 (80.6\%) patients had a clinically significant prostate cancer and 6/31 (19.3\%) had not significant cancer.

Cancer detection rate of mpMR was 18/31 (58.1\%), and 9/18 (50\%) for high grade tumors: sensitivity was $18 / 22$ (81.8\%) for all tumors and 11/19 (58.9\%) for high grade tumors.

Cancer detection rate inside ZSB/CTB was 14/25 (56\%) 
Figure 1.

Patient who underwent Zonal Saturation Biopsy. Mismatch between target area on transrectal ultrasound ( $A$, arrow) and the mpMRI in T2 (B, arrow on the ROI).

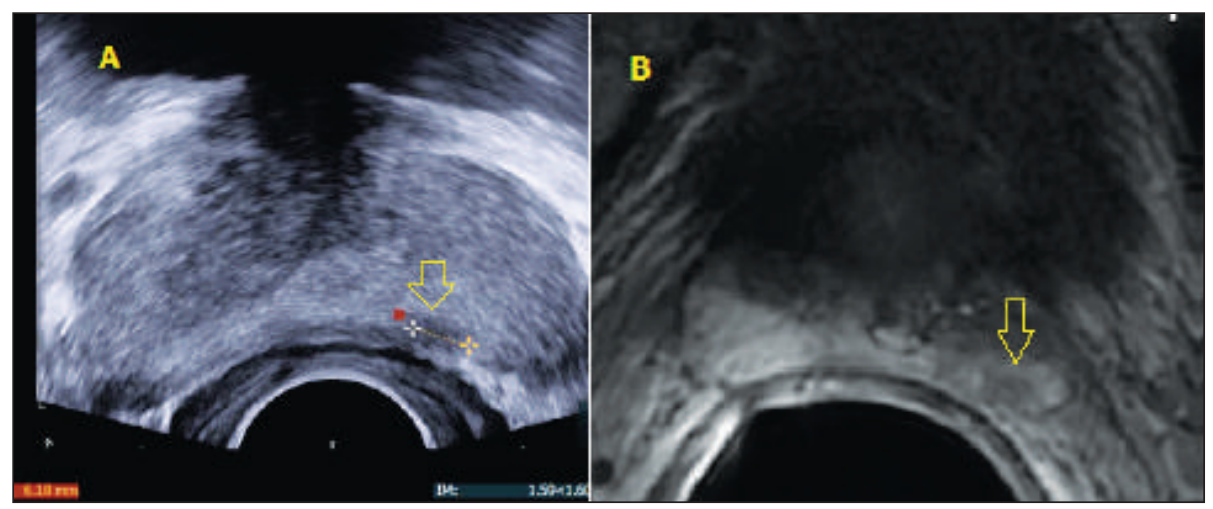

Figure 2.

Patient who underwent Cognitive Target Biopsy (CTB). Extact matching between target area on transrectal ultrasound ( $A$, yellow arrow) and the mpMRI in T2 (B, yallow arrow). Furthermore a midline cyst (red arrow) helps in the lesion using a spatial correlation with US and MRI.

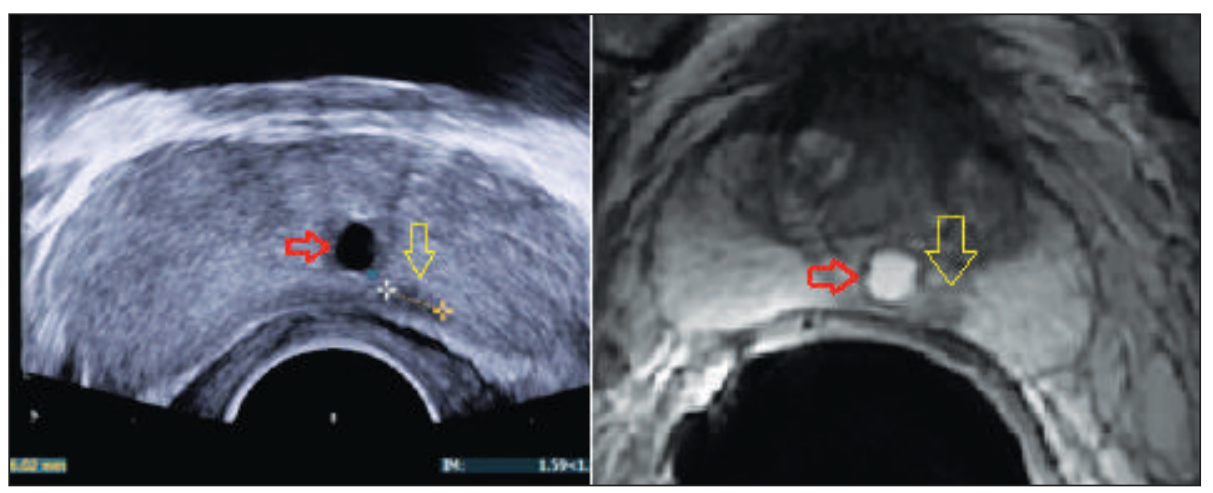

applied to a zone of the prostate containing the target lesion. Therefore applying this principles to daily practice, if a target lesion is evident comparing TRUS and MRI, we performed Cognitive Target Biopsy limited to the target using 2-4 cores in 26 $\%$. In prostate without ultrasound target lesion compared to MRI, the zonal saturation biopsy was performed in 74\% cases. Thus we supposed to reduce variability linked to cognitive fusion biopsy.

Whereas the pure cognitive biopsy may be extremely dependent on operator experience and imaging interpretation, the zonal saturation biopsy limits these factors causing a limited increase in number of cores $(+6$ in our experience).

Cognitive plays a key role in the decision-making process of therapeutic choice, because an increased detection rate of clinically significant cancer can drive the choice between active surveillance and surgery.

Based on our data, we can confirm that systematic 12 core biopsies in patients with negative or positive mpMRI should not be omitted. This

for clinically significant cancer and 4/6 (66.7\%) for not significant cancers. Outside the $\mathrm{ZSB} / \mathrm{CTB}$ results $5 / 13$ (38.5\%) for clinically significant cancer and 4/7 (57.1\%) for not significant cancers. Cancer detection rate was 8/17 (47\%) in PI-RADS 4/5 cancers, 16/36 (44\%) in PIRADS 3 and 0/5 in PIRADS 0-2.

The mean number of cores taken with cognitive zonal fusion biopsy was 6.1 (2-17), in addition biopsy sampling was done outside the ROI areas. Overall a mean of 15.4 cores (12-22) were taken. Mean core length was 1.5 $\mathrm{cm}$ (0.8-1.9) measured in histology slide.

Amount of cancer in Zonal Biopsy resulted $7.3 \mathrm{~mm}$ (154.5) in ZSB or CTB and $5.2 \mathrm{~mm}$ (1-23.5) outside ZSB or CTB. Cancer according to prognostic grading Group was grouped as 2-5 in 11/23 inside the ZSB/CTB and in $13 / 23$ outside the ZSB/CTB. Largest percentage of cancer involvement was detected in $19.44 \%$ (0-67.53\%) inside ZSB or CTB and $15.9 \%$ (0-50.7\%) outside ZSB or CTB.

\section{Discussion}

Cognitive zonal fusion biopsy may be considered an improvement of cognitive fusion biopsy. Cognitive zonal fusion is based on the principles of ultrasound target biopsy using end-fire probe and saturation biopsy may be valid in our area where the experience with mpMRI was not uniform.

In agreement with the study presented by Quentin et al. (3), TRUS-guided biopsy can be as good if done well. In 128 men, prostate cancer detection rate comparing inbore target MRI with TRUS guided biopsies was $60.9 \%$. When used alone, the MRI and TRUS biopsies had an identical detection rate (53.1\%) and a similar ability to detect clinically significant intermediate- and high-risk cancer (22 vs 23 patients). They did not find any significant difference in Gleason score of tumours diagnosed by the two methods. However, more than half of the lesions missed by TRUS-guided biopsy were located in the interior part of the prostate gland.

For tumors with equivalent Gleason grading, the mean tumor infiltration of biopsy cores was also greater with MRI biopsy (61.4\% vs 48.4\%; p <.0001).

Multiparametric MRI should be used to identify anatomical zones containing suspected lesions. ZSB is a technique useful in increasing the detection rate of clinically significant cancers in comparison to target biopsy. Zonal saturation has been done when the ultrasound is not enough to identify the suspected lesion found to mpMRI or any abnormal imaging was reported. In the few cases (5) with negative MRI an extended biopsy were done, 
without any evidence of tumor. MRI is the best tool we have to find clinically relevant cancer reducing overdetection, however MR has bias in interpretation and instrumentation leading to not uniform definition of PIRADS among different centers and operator.

Advantage of TRUS guided biopsy using end-fire probe over biplane probe has been validated $(7,8)$. Also significance of hypoechoic lesion on TRUS during cognitive fusion biopsy was recently outlined by Shkir et al. (8). Limitations of our study are bias in instrumentation and interpretation of MR, even if PI-RADS classification was used in different centers by several operators; in order to reduce this bias we included also suspect lesion (PIRADS 3). However the low detection rate observed for mpMR may reflect the "real life" value of a center with initial experience in mpMRI, such as in our region, rather than detection rate observed in referral centers with dedicated MRI. Another methodological limitation is based on limited number of cases and absence of a control group evaluated using MR/US fusion machine.

\section{Conclusions}

Cognitive zonal biopsy based on mpMRI findings plus standard extended biopsy identifies clinically relevant prostate cancer in $80 \%$. Cognitive zonal biopsy contains more cancer extension rather than random biopsies, and reduce the number of cores if compared to saturation biopsy. Systematic 12-core biopsies in patients with negative or positive mpMRI should not yet be omitted.

\section{REFERENCES}

1. Dickinson L, Ahmed HU, Allen C, et al. Magnetic resonance imaging for the detection, lo-calisation, and characterization of prostate cancer: recommendations from a European conensus meeting. Eur Urol. 2011; 59:477-94.

2. Barentsz JO, Richenberg J, Clements R, et al. ESUR prostate MR guidelines 2012. Eur Radiol. 2012; 22:746-57.

3. Quentin M, Blondin D, Arsov C, et al. Prospective evaluation of magnetic resonance imaging guided in-bore prostate biopsy versus systematic transrectal ultrasound guided prostate biopsy in biopsy naïve men with elevated prostate specific antigen. J Urol. 2014; 192:1374-9.

4. Galosi AB, Tiroli M, Cantoro D, et al. Biopsy of the anterior prostate gland: technique with end-fire transrectal ultrasound. Arch Ital Urol Androl. 2010; 82:248-252.

5. Bertaccini A, Fandella A, Prayer-Galetti T, et al. Italian Group for Developing Clinical Practice Guidelines on Performing Prostate Biopsy: Systematic development of clinical practice guidelines for prostate biopsies: a 3-year Italian project. Anticancer Res. 2007; 27:659-666

6. Scattoni V, Montironi R, Rigatti P, et al. Pathological changes of high-grade prostatic intraepithelial neoplasia and prostate cancer after monotherapy with bicalutamide $150 \mathrm{mg}$. BJU Int. 2006; 98:54-58.

7. Ploussard G, Aronson S, Pelsser V, et al. Impact of the type of ultrasound probe on prostate cancer detection rate and characterization in patients undergoing MRI-targeted prostate biopsies using cognitive fusion. World J Urol. 2014; 32:977-83.

8. Shakir NA, Siddiqui MM, George AK, et al. Should hypoechoic lesions on TRUS be sampled during MRI-targeted prostate biopsy? Urology. 2016.

\author{
Correspondence \\ Andrea B. Galosi, MD (Corresponding Author) \\ a.b.galosi@univpm.it galosiab@yahoo.it \\ Chief Institute of Urology, Marche Polytechnic University \\ Azienda Ospedaliera Universitaria "United Hospitals" - 60020 Ancona, Italy \\ Giulia Sbrollini, MD \\ giuliasbrollini@libero.it \\ Lorenzo Montesi, MD \\ lorenzomontesi@yahoo.it \\ Matteo Tallè, MD \\ matteo.talle@gmail.com \\ Resident, Institute of Urology, \\ Azienda Ospedaliera Universitaria "United Hospitals" - 60020 Ancona, Italy \\ Guevar Maselli, MD \\ guevarmaselli@katamail.com \\ Gaetano Donatelli, MD \\ gaetanodonatelli@hotmail.com \\ Division of Urology, "Murri" Hospital - 63900 Fermo, Italy \\ Rodolfo Montironi, MD \\ r.montironi@univpm.it \\ Institute of Pathology, Marche Polytechnic University \\ Azienda Ospedaliera Universitaria "United Hospitals" - 60020 Ancona, Italy
}

